\title{
Night-time image quality at Roque de los Muchachos Observatory
}

\author{
C. Muñoz-Tuñón ${ }^{1}$, J. Vernin ${ }^{2}$, and A.M. Varela ${ }^{1}$ \\ 1 Instituto de Astrofísica de Canarias, E-38200 La Laguna, Tenerife, Spain \\ 2 Département d'Astrophysique de l'Université de Nice, U.R.A. 709 du CNRS, F-06108 Nice Cedex 2, France
}

Received November 6, 1996; accepted January 2, 1997

\begin{abstract}
Nine months' seeing statistics at the Observatorio del Roque de los Muchachos (ORM) are presented. Measurements were taken with a differential image motion monitor on top of a 5-m high tower. From the whole campaign the mean seeing is $0.76^{\prime \prime}$, the median value is $0.64^{\prime \prime}$ and $7 \%$ of the time the seeing is better than $0.3^{\prime \prime}$. The best seeing measured is $0.17^{\prime \prime}$. A seasonal variation is noticeable, better seeing conditions appearing during summer, which corresponds to the occurrence of a well-defined inversion layer due to the high prevalence of trade winds. During the summer, $50 \%$ of the time seeing is better than $0.54^{\prime \prime}$, value which drops to $0.46^{\prime \prime}$ during June, with a narrow distribution $\left(\mathrm{rms}=0.17^{\prime \prime}\right)$. These figures place the ORM among, or better than, the best astronomical sites (Paranal and La Silla, Chile) where seeing statistics are available.

When inspecting individual nights, it sporadically happens that seeing deteriorates abruptly and slowly recovers, with a 1.2-hour exponential decay time. This phenomenon is interpreted in terms of meteorological instabilities (gravity waves) which trigger a turbulent regime.
\end{abstract}

Key words: atmospheric effects — site testing

\section{Introduction}

The Observatorio del Roque de los Muchachos (ORM), belonging to the Instituto de Astrofísica de Canarias (IAC), is located on the island of La Palma, one of the seven islands which constitute the Canarian archipelago. The Canaries are located around $28^{\circ} \mathrm{N}$ latitude and $17^{\circ} \mathrm{W}$ longitude, $1300 \mathrm{~km}$ south to the European continent. The climatology of the Canary Islands has been studied in great detail through long data series (Font-Tullot 1956; Huetz de Lemps 1969). The main characteristic which defines their excellence as observing sites is the stability of the

Send offprint requests to: C. Muñoz-Tuñón height of the temperature inversion layer, typically below $1500 \mathrm{~m}$. Being situated at $2400 \mathrm{~m}$ above sea level on an isolated peak, the ORM benefits most of the time from dry, stable and transparent atmosphere (Ardeberg 1984; Murdin 1985; Muñoz-Tuñón \& Fuentes 1991).

Since the last century, there have been many studies of the quality for astronomical observations both at the ORM and the Observatorio del Teide (Tenerife). A very comprehensive review from the beginning up to 1985 can be found in Murdin (1985). Much other useful information can also be found in this issue of Vistas, which is entirely dedicated to the observatories of the Canary Islands.

This paper is devoted to night-time observations. Daytime site-testing campaigns have also been carried out at the Canaries. Among these we can mention the JOSO (Joint Organization for Solar Observations, Brand \& Righini 1985) and, more recently, the LEST (Large Earthbased Solar Telescope) campaigns.

A key parameter in determining how good is an astronomical site is is surely the image quality (see Coulman 1985, and references therein). In the last few decades, a huge effort has been devoted to the development of new-generation large-diameter telescopes and to the implementing of very sophisticated post-focus instrumentation which require a very precise site characterization. In this sense, since the pioneering work by ESO in Chile, "new philosophy" intensive site-testing campaigns (Vernin \& Muñoz-Tuñón 1992) have been performed at candidate sites and in already existing observatories to verify whether they matched the necessary criteria for these new-generation astronomical facilities. Since 1985, site assessment campaigns have been carried out by some of the authors at Mount Locke (Texas), La Silla and Paranal (Chile), Mauna Kea (Hawaii) and the ORM (La Palma). The main aim of these campaigns was to assess, by means of a variety of simultaneous experiments (see e.g. Vernin \& Muñoz-Tuñón 1992), the vertical profile of $C_{\mathrm{N}}^{2}$ and therefore the relative contribution and identification of individual turbulent layers (Vernin \& Muñoz-Tunón 1994; Coulman et al. 1995). The measurement of simultaneous 
other atmospheric parameters, such as temperature, humidity and wind, are of major importance in the interpretation of the occurrence of optical turbulence.

Many different attempts have been made to measure seeing since the pioneering work by Rösch (1963). Although the parameters relevant to image degradation are related to geophysics (turbulent fluctuations of air density), all seeing monitors are optical instruments; balloon data (Vernin \& Muñoz-Tuñón 1992, 1994) can be used but cannot monitor it versus time. Different concepts have been used: the Danjon method, Polaris trails, shearing interferometers (Roddier 1976), the Scidar technique (Azouit \& Vernin 1980), differential image motion monitors (DIMMs) (Stock \& Keller 1960; Sarazin \& Roddier 1990), the grating scale monitor (Martin et al. 1994). These use different receivers such as the eye, photographic plates, photomultipliers or intensified CCDs. To monitor the seeing, the Département d'Astrophysique (DA) of the University of Nice and the Instituto de Astrofísica de Canarias (IAC) developed a DIMM based on a principle first proposed by Stock \& Keller (1960). The DA/IAC DIMM was cross-calibrated with other existing ESO DIMMs during a joint campaign at Mount Paranal, Chile. The results from this comparison were excellent (Vernin \& Muñoz-Tuñón 1994). A very comprehensive description of the fundamentals and capabilities of the instrument can be found in Vernin \& MuñozTuñón (1995).

Although many new instruments and many intensive site-testing campaigns have been developed in the recent years, very little long-term seeing climatology is available yet. Even very good observatories, such as Mount Graham or Hawaii, lack of this kind of database, which nevertheless seems imperative for a proper site comparison to be done. Only La Silla and Paranal, and now the ORM (present data) have systematic seeing measurements taken with cross-calibrated instruments.

In this paper, we analyse seeing data taken at the ORM with two DA/IAC DIMMs. In Sect. 2 there is a brief summary of the DIMM principle along with the data aquisition and processing. In Sect. 3 the results of the statistical analysis is described. In Sect. 4 two different areas at the ORM are compared. In Sect. 5 the temporal dependence of seeing values is studied, and the summary and final remarks are given in Sect. 6 .

\section{Experiment and measurements}

The DIMM principle is to produce twin images of a star with the same telescope via two entrance pupils separated by a distance $d$ in order to eliminate erratic motions of the telescope. The knowledge of the phase structure function, which is assumed to result from Kolmogorovian turbulence, enables us to assess the longitudinal and transverse (parallel and perpendicular to the aperture aligment) variance of differential image motions as given by

$\sigma_{l ; t}^{2}=\sigma^{2}\left(1-k_{l ; t}\left(\frac{D}{d}\right)^{\frac{1}{3}}\right)$,

with $k(l)=0.541$ and $k(t)=0.810$, which holds when $D / d$ $\leq 0.5$ (see e.g. Sarazin \& Roddier 1990). The variance and the Fried parameter $\left(r_{0}\right)$ are related by

$$
\left.\sigma^{2}=0.358\left(\frac{\lambda}{r_{0}}\right)^{\frac{5}{3}}\left(\frac{\lambda}{D}\right)^{\frac{1}{3}}\right)
$$

From the above relations two independent $r_{0}$ values are obtained. From $r_{0}$, classical astronomical seeing $\left.\left(\varepsilon_{\mathrm{FWHM}}\right)\right)$ is obtained through $\varepsilon_{\mathrm{FWHM}}=0.98 \lambda / r_{0}$ (Dierickx 1992). To simplify the nomenclature we will hereafter refer to this as FWHM or seeing.

The DA/IAC DIMM consists of an 8-inch Celestron, an equatorial mount, a pulsed intensified CCD camera and an image grabber (MATROX) connected to a PC computer. A mask made of two diaphragms (60-mm diameter) is located at the entrance pupil of the telescope, and a prism (of about $30^{\prime \prime}$ deflection) is placed over one of them to produce two images. To match the pixel size, the focal length is enlarged using an eyepiece with an optical gain $G \approx 3$. The twin images of a star are captured by the intensified camera with a exposure time $\Delta T \approx 10 \mathrm{~ms}$, short enough to freeze the wave-front. The video signal is then digitized.

Seeing is referred to zenith taking into account the appropriate air-mass correction, the zenithal angle being small $\left(\leq 30^{\circ}\right)$. FWHM is accurate to better than $0.1^{\prime \prime}$ and, under reasonably good conditions it is possible to monitor the seeing with a temporal sampling rate of less than half a minute for several hours, thanks to the automatic guiding.

Seeing data to be analysed in this paper have been obtained at two sites at the ORM, one near the location of the Telescopio Nazionale Galileo (TNG) (hereafter referred to as site A) and a second one close to the William Herschel $(4.2 \mathrm{~m})$ Telescope (WHT, site B). In Fig. 1 the location of sites A and B are marked on a portion of the ORM map. The locations of three telescopes, the WHT, the TNG and the Nordic Optical Telescope (NOT), used as reference points, are also indicated.

Routine measurements were carried out 3 to 4 nights every week. To avoid the surface layer (SL) influence (Vernin \& Muñoz-Tuñón 1994), the DIMM at site A was located on a 5-m tower designed by the Galileo Project. The DIMM situated on site B belongs to Isaac Newton Group and was installed on top of the WHT terrace.

As mentioned previously, both longitudinal and transverse FWHM are recorded and subsequently used to determine the validity of the measurements and to improve them. During an observing run, when a systematic discrepancy is noticed between $\mathrm{FWHM}_{l}$ and $\mathrm{FWHM}_{\mathrm{t}}$ of more than $12 \%$, which is the relative error expected from the 


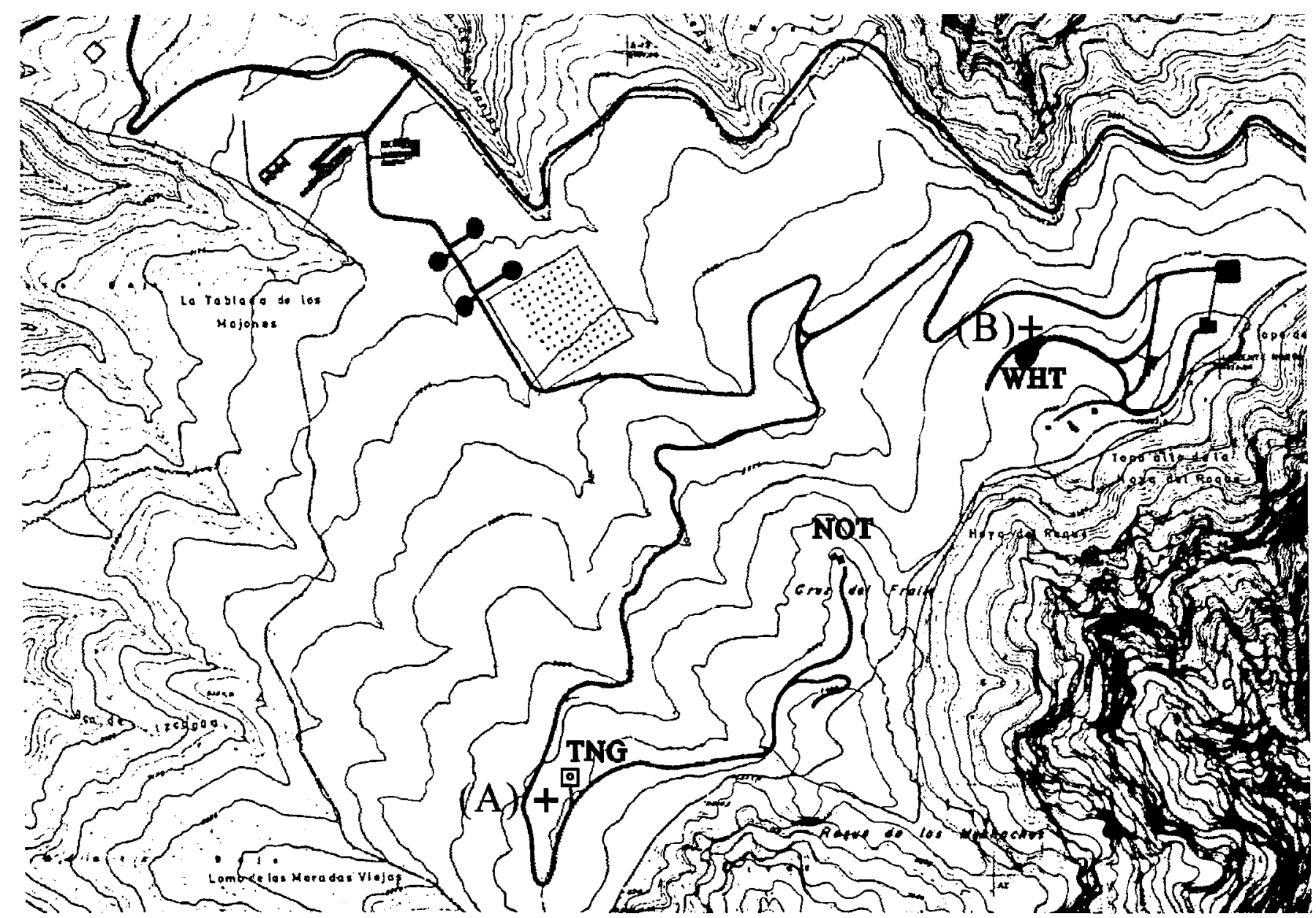

Fig. 1. Map of the ORM showing the location of sites A and B. The location of the Telescopio Nazionale Galileo (TNG), the Nordic Optical telescope (NOT) and the William Herschel Telescope (WHT) are also indicated for reference. The volcano rim of the Caldera de Taburiente flanks the observatory to the south

Table 1. Data corresponding to seeing mesurements at site A (ORM near the TNG site). For each month the number of seeing measurements is given. The statistics of each month, minimum, mean, standard deviation and median values of seeing in arcseconds is presented

\begin{tabular}{|c|c|c|c|c|c|}
\hline month & Ndata & min $\left(^{\prime \prime}\right)$ & mean $\left(^{\prime \prime}\right)$ & std $\left(^{\prime \prime}\right)$ & median $\left(^{\prime \prime}\right)$ \\
\hline $11-94$ & 6744 & 0.26 & 0.81 & 0.34 & 0.74 \\
$01-95$ & 11957 & 0.23 & 0.94 & 0.64 & 0.77 \\
$02-95$ & 10582 & 0.2 & 0.95 & 0.44 & 0.84 \\
$03-95$ & 3793 & 0.32 & 1.13 & 0.87 & 0.77 \\
$04-95$ & 9119 & 0.26 & 0.82 & 0.5 & 0.66 \\
$05-95$ & 11906 & 0.17 & 0.74 & 0.37 & 0.64 \\
$06-95$ & 11724 & 0.20 & 0.49 & 0.17 & 0.46 \\
$07-95$ & 9675 & 0.19 & 0.61 & 0.26 & 0.56 \\
$08-95$ & 10462 & 0.22 & 0.60 & 0.23 & 0.54 \\
\hline all & 85962 & 0.17 & 0.76 & 0.47 & 0.64 \\
\hline
\end{tabular}



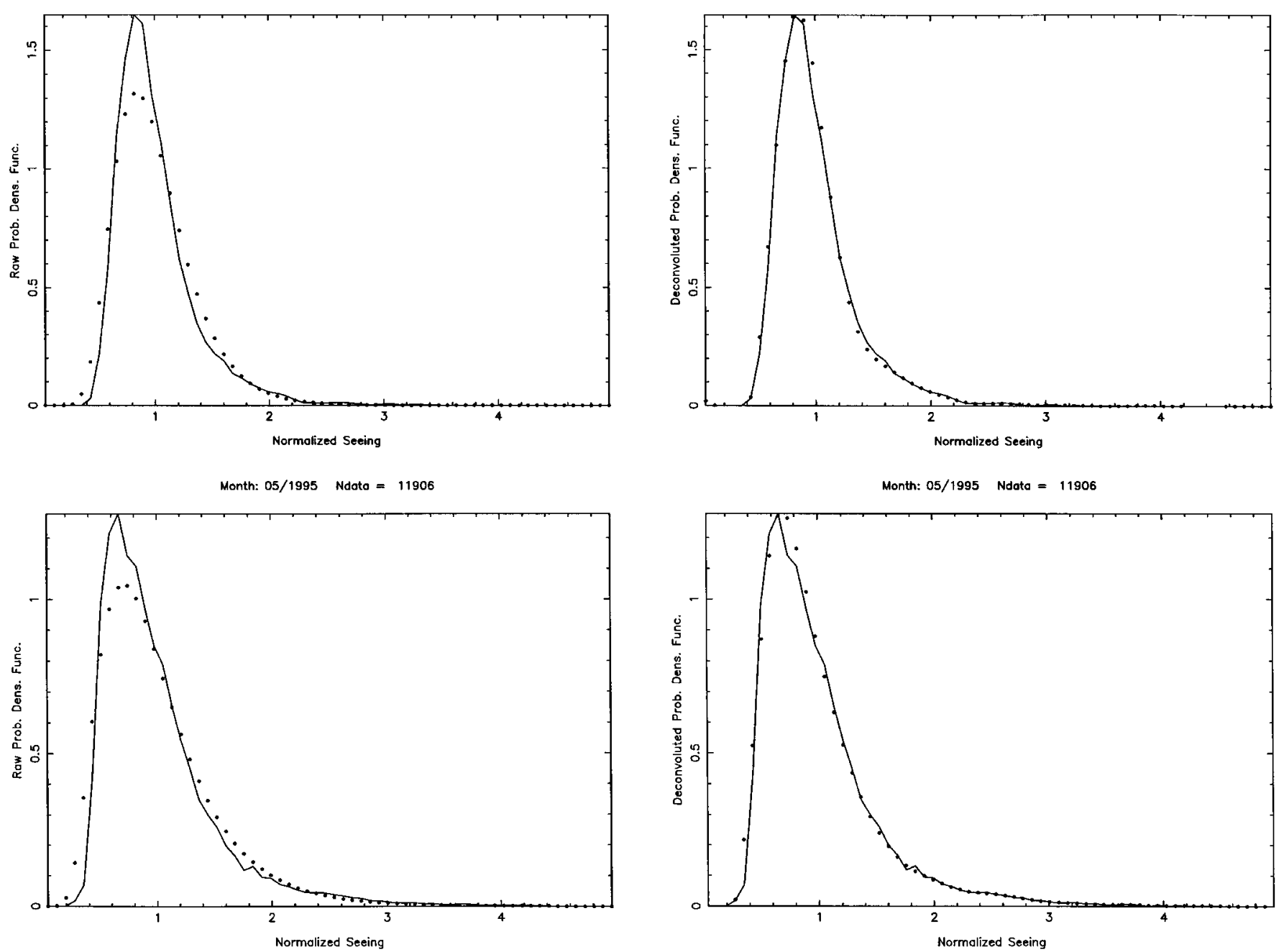

Fig. 2. The distribution function of measured seeing corresponding to two months at site A is shown by a continuous line. The dotted line (left) refers to the deduced log-normal distribution resulting from the measurement of the second moment. The dotted line (right) represents the result of the deconvolution assuming an additive Gaussian noise

number of images processed and other noise (see Vernin \& Muñoz-Tuñón 1995), the data are discarded, otherwise they are averaged.

The distribution function of a positive random variable, like seeing, is expected to be log-normal (see e.g. Lee 1960). In Fig. 2 (left) we display with a solid line the distribution function of measured seeing for two different months, along with the deduced log-normal distribution (dotted line) one would expect from the measurement of the second moment. It is clear that the deduced log-normal distribution is broader and smoother compared to the observed one. We already know that there is spurious noise, due to the low number of images processed to provide an individual seeing measurement $(N=200)$, which certainly may affect the shape of the distribution function.

Let us suppose that this noise is additive and does not depend on the seeing value; then the probability density function (pdf) of the measured seeing, $P_{\mathrm{m}}(x)$, is the convolution of the true pdf of the seeing, $P_{\varepsilon}(x)$, and the noise pdf, $P_{\text {noise }}(x)$ :

$P_{\mathrm{m}}(x)=P_{\varepsilon}(x) * P_{\text {noise }}(x)$.

A simple deconvolution is made assuming a Gaussian behaviour of the noise. In Fig. 2 (right) we plot with a dotted line the deconvolved pdf, evaluating an rms noise compatible with the number of processed images. One notes the fit improvement which proves that our seeing data are compatible with an expected log-normal distribution of the seeing and a Gaussian additive noise. 

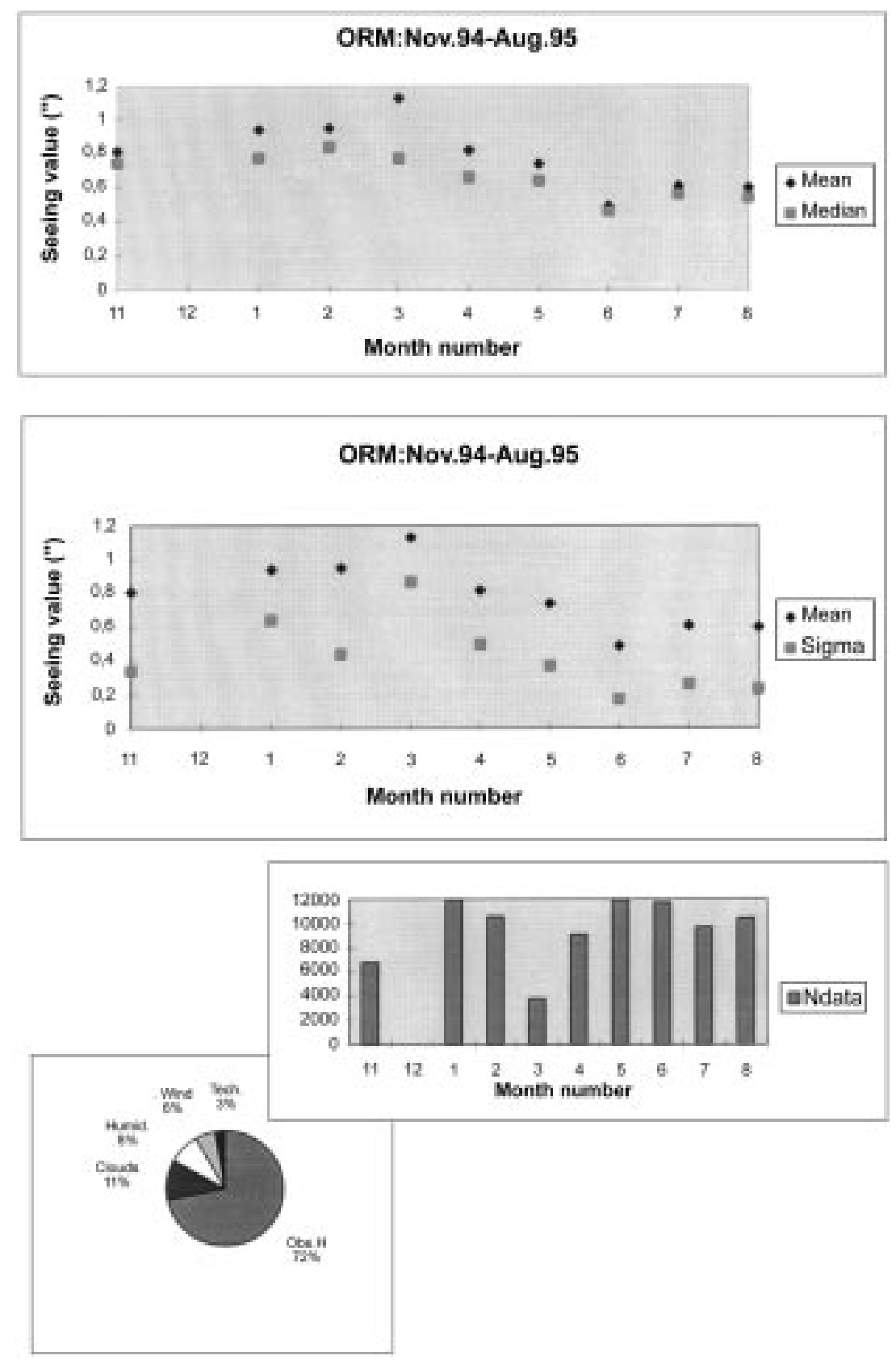

Fig. 3. Mean, median, minimum and rms seeing values for each month at site A. The statistic of observed time with the seeing monitor during the campaign at site $\mathrm{A}$ is plotted

\section{Statistical results}

The high image quality at the ORM is now verified throughout an extensive statistical database of reliable seeing values.

In Fig. 3, results corresponding to 9 months of measurements at site A are presented. The lack of data for December 1995 is due to instrumental problems (coma distortion in the telescope). For each month, the statistical parameters obtained from the seeing value distribution are calculated. The monthly trend of mean, median, mini- mum and the standard deviation is shown. In order to give an idea of the statistical significance of the values the total number of observations taken every month is presented as well as the percentage of observed hours with the seeing monitor. The corresponding numerical values are given in Tables 1 and 2 .

As is evident in Fig. 3 and Tables 1 and 2 the monthly median seeing never reaches values worse than $1^{\prime \prime}$. The minimum atmospheric degradation to image quality can be measured throughout the lowest seeing values (also 
presented in Fig. 3). In this sense it may be pointed out that seeing below $0.3^{\prime \prime}$ is achieved in all sampled months except March.

From our measurements with the DIMM we estimate $72 \%$ of observed hours from the available observing time, $11 \%$ being lost due clouds, $6 \%$ due to winds higher than $15 \mathrm{~m} \mathrm{~s}^{-1}$, which is the limit of the instrument, and $8 \%$ due to high humidity (misting up of the optical surfaces). This percentage could be considered as a pessimistic estimate of useful observing time at the ORM. Given the fact that our instrument operates in the open air, it is more affected by air humidity changes than other astronomical telescopes. Therefore in this sense we certainly are more limited in the useful hours. Also, the instrument is very sensitive to the presence of even tenuous clouds. Cirrus-like clouds are detected by the DIMM, with the consequent loss of tracking of the source. So with the above-mentioned provisos, the results we obtain are in very good agreement with those reported from the Carlsberg Automatic Meridian Circle (CAMC), taken over several years and compiled by Sarazin (1995), which indicate $79 \%$ of useful observing time at the ORM.

\subsection{Seasonal dependence of seeing}

A possible seasonal dependence of seeing at the Canarian observatories has been the subject of discussion for a number of years. Sanchez (1970) found a slight tendency for nights of very good seeing at Izaña (Tenerife) to occur in summer between April and October (1962-1966). At La Palma, Pike (1984), from data taken at the telescope focus, found the summer months to show an average seeing better than during the winter. The accuracy of the seeing monitor together with the temporal sampling of data presented here make them extremely useful in deciding this question.

From Fig. 3 it is clear that the seeing abruptly improves between May and June. In Fig. 4 it is shown results from a comprehensive study of the climatology of the Canary Islands (Font-Tullot 1956). In this plot, using data covering 20 years compiled by the Instituto Nacional de Meteorología at Tenerife, the annual variation of the scale height of the sea-cloud (inversion layer) is shown.

The existence of a temperature inversion layer is a characteristic of subtropical regions, and in the Canaries it is registered about $90 \%$ of the time. The efficiency of the inversion layer in separating the boundary layer from the troposphere is clear on the humidity profile, where typically $55 \%$ of the humidity is situated below the inversion and $20 \%$ above it (Cuevas 1995). The inversion suppresses local convection, which is clearly visible in the presence of a characteristic stratocumulus layer whose top is just below the temperature inversion (Font-Tullot 1956). The frequency of occurrence of the cloud layer is maximum in summer and is anti-correlated with its height (Fig. 4).
Table 2. Data corresponding to seeing mesurements at site A (ORM near the TNG site). For each month, the number of available hours, of observed hours, of hours lost due to clouds, humidity, wind and technical problems are given

\begin{tabular}{|c|c|c|c|c|c|c|}
\hline date & av. h. & obs. h. & cl. & hum. & w. & t.p. \\
\hline $11-94$ & 168. & 86. & 59.5 & 0.0 & 20. & 2.5 \\
$01-95$ & 180. & 143. & 6. & 0.0 & 24. & 7. \\
$02-95$ & 126.5 & 115.0 & 2.0 & 9.5 & 0.0 & 0.0 \\
$03-95$ & 176.0 & 46.9 & 25.5 & 79.6 & 24.0 & 0.0 \\
$04-95$ & 143.0 & 106.5 & 6.5 & 28.0 & 2.0 & 0.0 \\
$05-95$ & 160.0 & 144.0 & 10.5 & 0.0 & 5.5 & 0.0 \\
$06-95$ & 123.0 & 123.0 & 0.0 & 0.0 & 0.0 & 0.0 \\
$07-95$ & 110.0 & 106.0 & 4.0 & 0.0 & 0.0 & 0.0 \\
$08-95$ & 120.0 & 112.0 & 8.0 & 0.0 & 0.0 & 0.0 \\
\hline
\end{tabular}

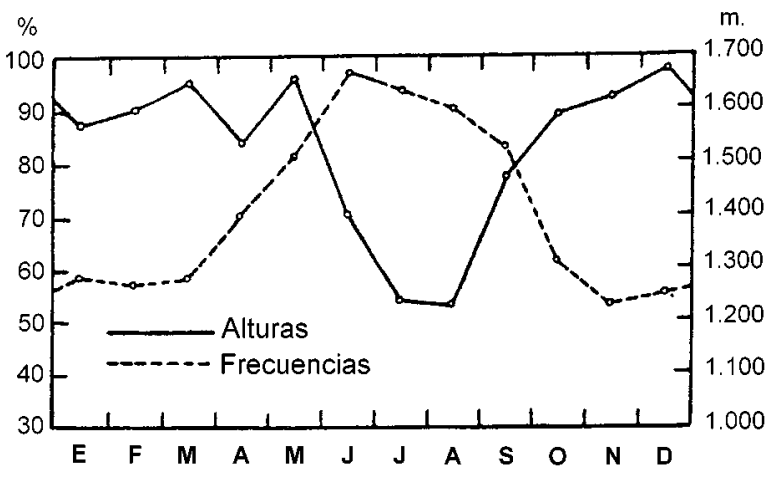

Fig. 4. Annual variation of the scale height and frequency of the sea clouds in Tenerife. Taken from Font-Tullot (1956)

The width of the inversion layer is greatest during the summer period.

The strength of the inversion layer (IL) is defined by the difference of temperature between the top and the base of the temperature inversion, and is maximum during the summer, as is the intensity of the trade-wind regime. The strong temperature inversion layer, and therefore the presence of the sea clouds, is caused by two complementary processes, the trade winds blowing from the NE carrying wet and cool air masses and the subsidence from the NW which occurs above the IL transporting dry air (Cuevas et al. 1996).

Despite the fact that the inversion layer tends to be well below the observatory location, it seems that a dependence of image quality exists, which is correlated with the height of the inversion layer (the sea-cloud location). The best seeing values seem to correspond to months where the altitude of inversion layer is lower than normal (in the $1500-1700 \mathrm{~m}$ range), or when the strength of the IL is maximum, which in turn is related to the strength of the trade wind regime.

In Fig. 5 seeing data are grouped following the above discussion in two blocks, one including June, July and 


\section{$\begin{array}{lll}\text { O.R.M. Summer Period } & \text { Nata }=43767 & \text { 4. Comparison of the TNG and WHT slopes }\end{array}$}

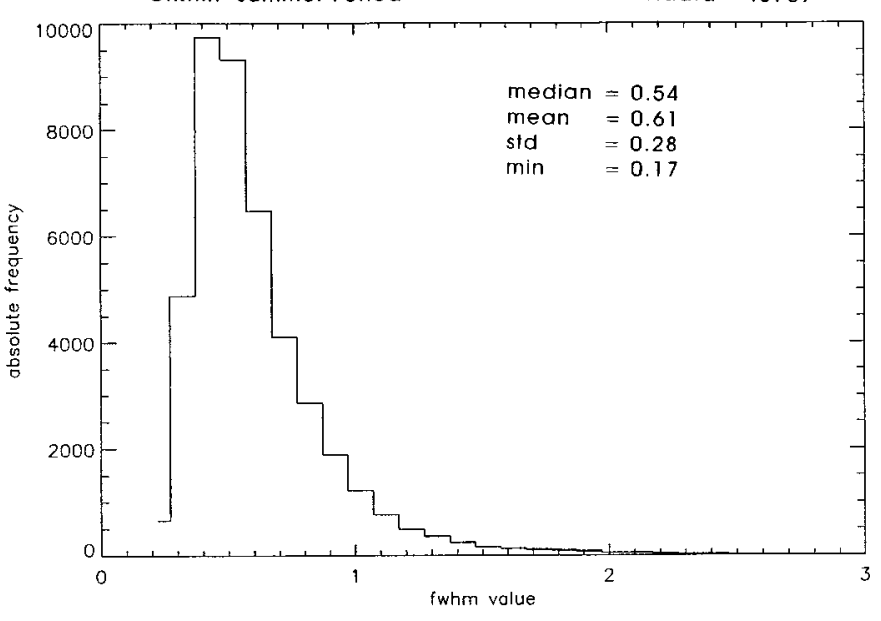

The building of a large telescope at the ORM is a great challenge for the Spanish astronomical community. The project was first promoted by the IAC and the Canarian Government and therefore we shall refer to it as the Gran Telescopio Canario (GTC). This national project has been widened in anticipation of the possible participation of important foreign astronomical institutions (RodriguezEspinosa \& Alvarez 1996). Some of the authors are in charge of defining the future location of the GTC. A detailed programme is now in progress towards the taking of the decision of where to install the GTC at the ORM.

An imaginary line drawn between the NOT and the Residence divides the ORM into two slopes (see Fig. 1). There are more observing installations on the northern-

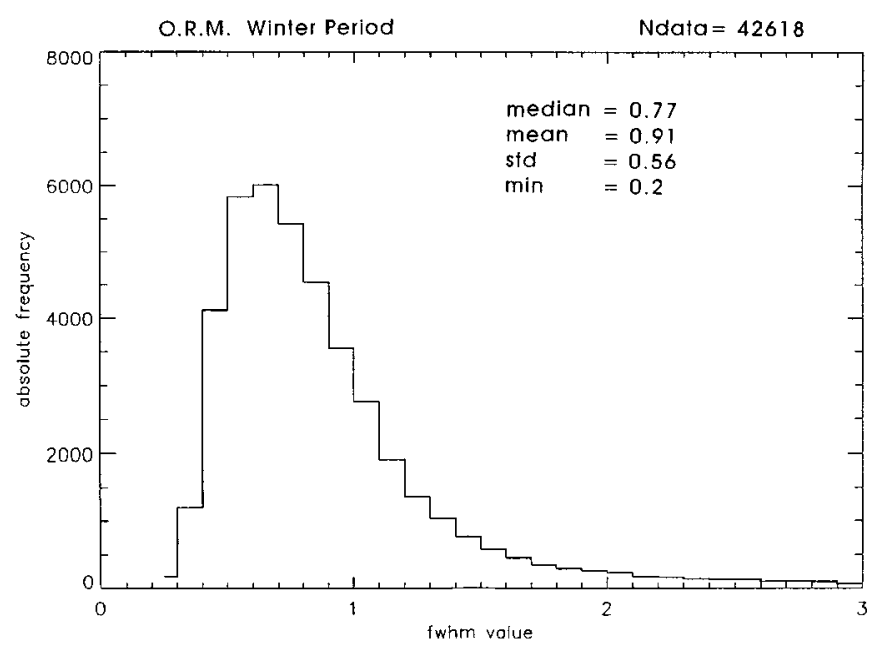

Fig. 5. Comparison of the statistics corresponding to winter and summer at site $\mathrm{A}$

August (summer) and the other containing the rest.The distribution function corresponding to summer gives better mean and median values and smaller stardard deviation. In Fig. 6 (top), the seeing distribution and the cumulative distribution function corresponding to June 1995 (best seeing) at site $\mathrm{A}$ are shown. The attained values are remarkable. The seeing is better than $0.5^{\prime \prime}$ for $60 \%$ of the time and better than $0.3^{\prime \prime}$ in $7 \%$ of cases. The mean and median values are very similar $\left(0.49^{\prime \prime}\right.$ and $0.46^{\prime \prime}$, respectively) with $0.17^{\prime \prime}$ stardard deviation. These values are very good even when compared with other astronomical sites known for their excellent seeing (see Sarazin 1995 for references). In Fig. 6 (bottom) the statistics corresponding to all data taken at site $\mathrm{A}$ are shown. Half of the time seeing values are better than $0.64^{\prime \prime}$ with minimum values down to $0.17^{\prime \prime}$. most side of that line. Hereafter this side will be referred to as the WHT slope. On the southernmost side there were no installations until the early $90 \mathrm{~s}$, when the Galileo group chose this area as the site for the TNG. This will be referred to as the TNG slope.

As mentioned in Sect. 1 the WHT slope has been extensively studied, due to the long-standing presence of large facilities and instruments. The NOT location has also been recognized as an excellent site through numerous studies carried out as well as through astronomical results taken with the telescope.

Following the statistics presented in Sect. 3 at site A on the TNG slope, there is no doubt concerning the very good image quality that can be achieved in this area of the ORM, which would be the more suitable one for the installation of new telescopic facilities. However a comparison of the two slopes with standard techniques was imperative.

Two identical seeing monitors were operated simultaneously: the DIMM at site B (Fig. 1) near the WHT belongs to the ING (Isaac Newton Group) and was operated by the IAC group during the period corresponding to data shown here. The data acquisition procedure and statistical processing is the same for both sites $\mathrm{A}$ and $\mathrm{B}$ and the results are presented in Table 3.

In comparing Tables 2 and 3 , it is seen that, to within the errors, the data are quite similar at both sites during the winter period. In the summer period, however, the seeing recorded at site A is better. This could be understood as due to local disturbances that might affect the measurements at site B. As mentioned before, the DIMM on site B is located on the WHT roof, which might release more heat during the night in summer than in winter. For what concerns seeing, both sites look very similar, which means that, except the possible disturbance of WHT terrace, the surface layer has a small (Vernin \& Muñoz-Tuñón 1994) and uniform contribution whatever the position within the ORM. 
O.R.M. -LA PALMA; NDATA $=11724$
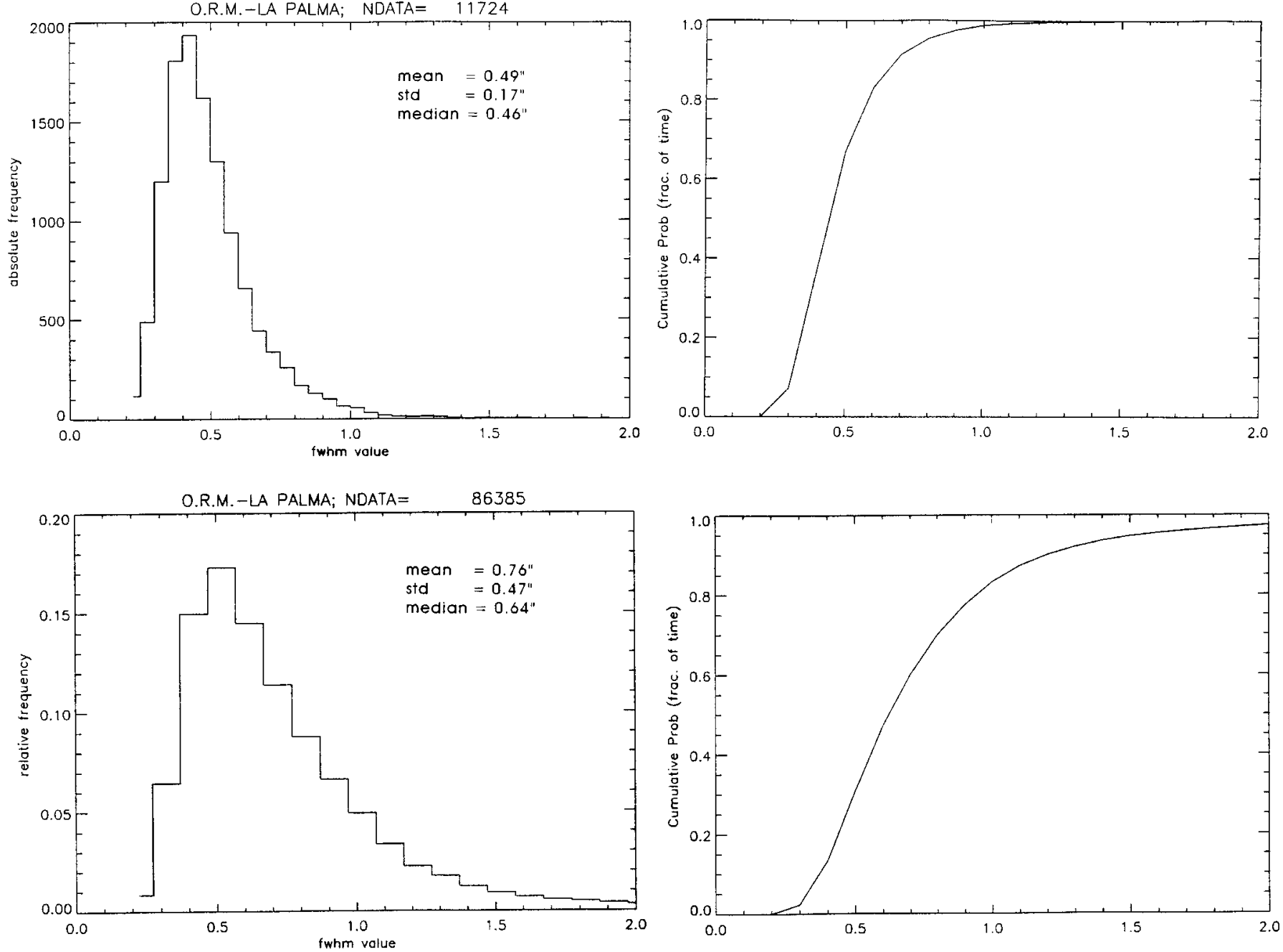

Fig. 6. (Top) seeing distribution and cumulative distribution function corresponding to June 1995 at site A. (Bottom) seeing distribution and cumulative seeing distribution from all data taken at site A

\section{Temporal dependence}

We have analysed the temporal variation of the seeing in order to have a better insight into the atmospheric behaviour which gives rise to optical turbulence. Recently a number of phenomenological processes have been described (Coulman et al. 1996). In this paper, it is assumed that optical turbulence is concentrated in twin laminae (very thin turbulent layers $\approx 10 \mathrm{~m}$ ) which appear on top and at the bottom of a unique thick mechanical turbulent layer $(\approx 300 \mathrm{~m})$. The authors of that paper suppose that in the greater part of the atmosphere the critical Richardson number $\left(R_{i}<\frac{1}{4}\right)$ is not attained and that turbulent conditions are triggered by localized gravity waves which appear suddenly and then propagate with oscillatory behaviour.

From the point of view of astronomers it would be convenient to be able to have a characterization of the temporal evolution of the seeing, i.e. to know what the typical time interval of seeing variation is, and also the dependence of seeing quality with time, if any. With this information one can conceivably optimize astronomical facilities. Up to now no general laws have been established.

In Fig. 7 the result of averaging all observing nights is shown versus UT and no general trend in the seeing evolution is observed. This is an important conclusion which can be opposed to the generally accepted assumption that "seeing is worst at the beginning of the night". Our measurements are free of local disturbances that occur in telescope buildings which, in the best cases, reach thermal equilibrium after several hours.

Inspecting individual nights, it can be observed that during relatively stable nights, with good seeing $\left(0.5^{\prime \prime}\right.$ typical mean values), the seeing can deteriorate over short periods. One can clearly see, as is shown in Fig. 8, in the middle of the night, a steep rise in the seeing from less than $0.5^{\prime \prime}$ up to more than $2^{\prime \prime}$ in less than 10 minutes. 
Table 3. Data corresponding to seeing measurements at site B (ORM near the WHT)

\begin{tabular}{|c|c|c|c|c|}
\hline date & median & mean & std & min \\
\hline $11-94$ & 0.78 & 0.84 & 0.37 & 0.20 \\
$01-95$ & 0.81 & 0.90 & 0.41 & 0.25 \\
$02-95$ & 0.96 & 1.09 & 0.55 & 0.27 \\
$03-95$ & 0.69 & 0.98 & 0.68 & 0.23 \\
$04-95$ & 0.64 & 0.72 & 0.32 & 0.21 \\
$05-95$ & 0.63 & 0.74 & 0.43 & 0.15 \\
$06-95$ & 0.58 & 0.66 & 0.33 & 0.17 \\
$07-95$ & 0.65 & 0.74 & 0.40 & 0.11 \\
$08-95$ & 0.72 & 0.88 & 0.49 & 0.21 \\
\hline all data & 0.69 & 0.81 & 0.45 & 0.11 \\
\hline
\end{tabular}

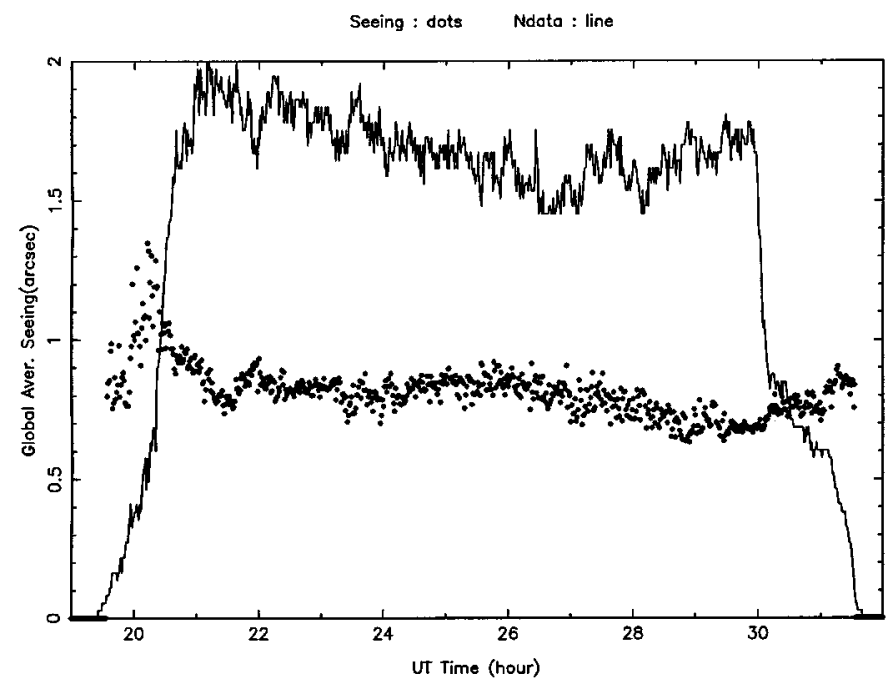

Fig. 7. Averaged seeing versus UT for the entire observing period at site A (nine months)

After this burst, one or two hours are necessary for good image quality to be recovered. This can be interpreted by saying that even under good seeing conditions and hence quasi-laminar flow, the steady equilibrium can be broken by a perturbation and degenerate into turbulence within a few minutes. These assumptions are supported by the fact that we never noticed an abrupt seeing improvement (the sequence is not reversible).

Another pattern that may occasionally appear in the temporal evolution of seeing is shown in Fig. 9 in which an oscillatory behaviour is observed. Also in this figure the corresponding autocorrelation function is shown for time intervals ranging from $\Delta t=0$ to $4 \mathrm{hr}$. The plot of Fig. $9 \mathrm{~b}$ ends at $\Delta t=4 \mathrm{hr}$. For longer time intervals, the number of measurements during a single night is not sufficient for the autocorrelation function to make sense. From Fig. $9 \mathrm{~b}$ a 45 -min periodic variation is visible. Superimposed on this oscillation, one can note also a slow decrease and after a 3-hour delay no correlation remains.

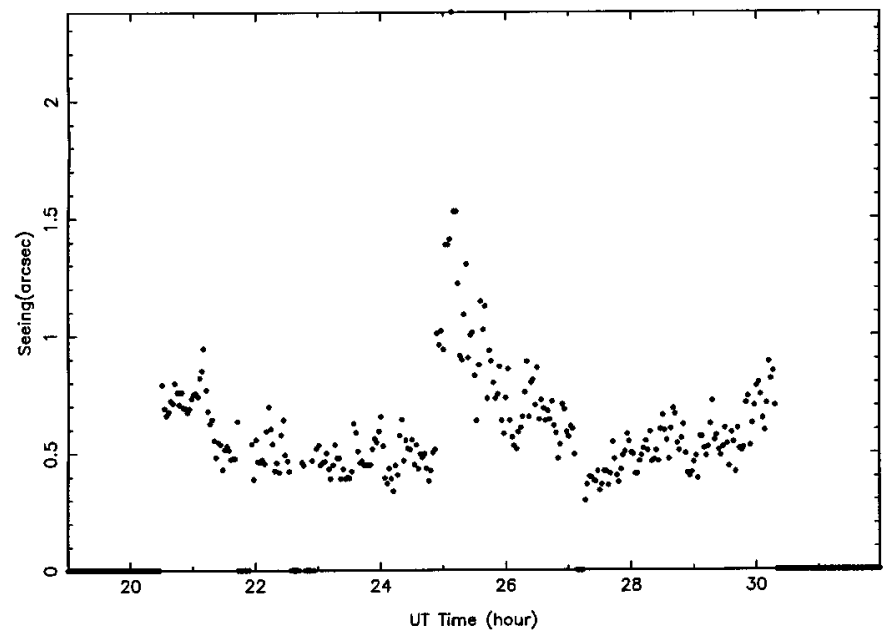

Fig. 8. Seeing versus UT on the night of 1995 May 4 at site A

As pointed out previously, the presence of longer timeinterval correlations cannot be studied through singlenight observations. Moreover we are also interested in the possible presence of a universal temporal behaviour of seeing to be interpreted in more general theories concerning turbulence phenomena. For so doing we analysed the averaged autocorrelation function, computed over all the available data during the nine months (Fig. 10). We have discarded the nights with less than 6 hours of observations. Each individual daily correlation has been centred and normalized to the mean seeing:

$C_{\varepsilon}(\tau)=<\frac{<\varepsilon(t) \varepsilon(t+\tau)>_{i}-<\varepsilon>_{i}^{2}}{<\varepsilon>_{i}^{2}}>$,

where $i$ index refers to individual night. No oscillations seem to remain in the correlation and therefore a large spectrum of (gravity) waves might be present in the atmosphere. For small time delay, the correlation closely follows an exponential decay with a $T=1.2$-hr time constant.

$C_{\varepsilon}(\tau)=A \exp (-\tau / T)$

with $A=0.071$.

As mentioned before, if one assumes that the seeing is the superimposition of many individual exponential decay functions of the type

$\varepsilon_{i}(t)=\mathrm{e}^{-\left(t-t_{i}\right) / T}$

for $t \geq t_{i}$ and 0 otherwise, then the autocorrelation of such a temporal decay is

$\int_{i} \varepsilon_{i}(t) \varepsilon_{i}(t+\tau) \mathrm{d} t=\mathrm{e}^{-\tau / T}$.

The best fit between our data and the above-mentioned assumption leads to a 1.2-hour time scale of decay of turbulence. 

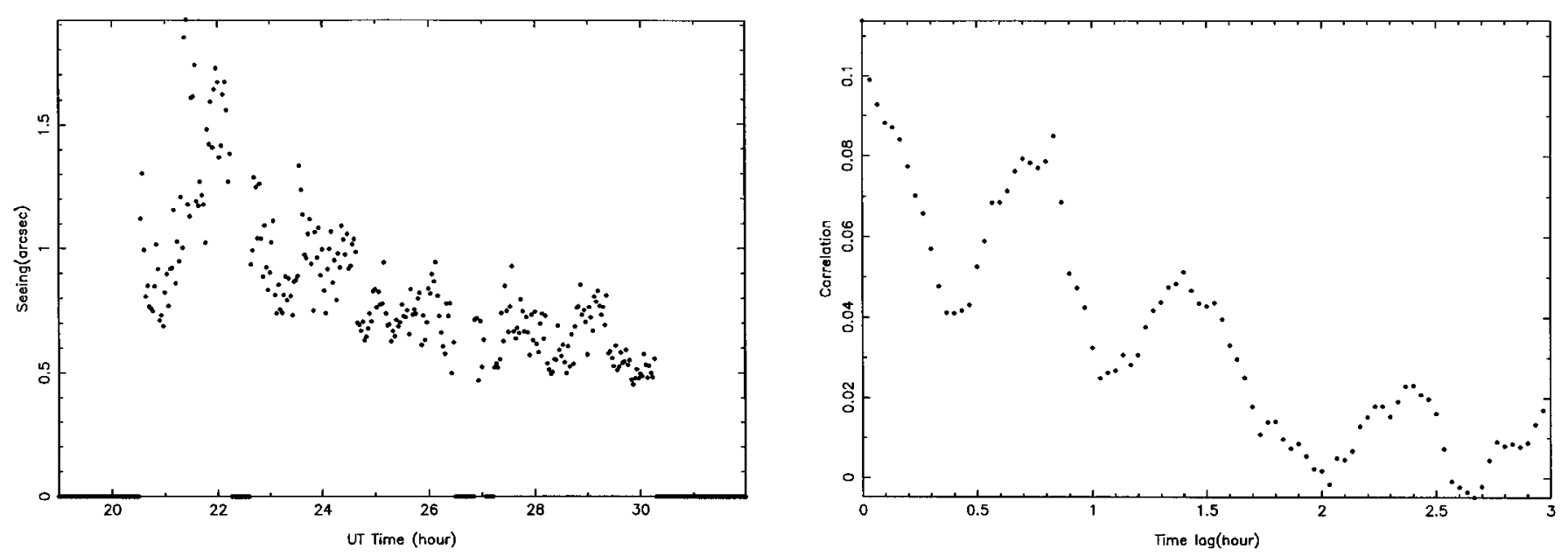

Fig. 9. a) Seeing versus UT on the night of 1995 May 10 at site A; b) autocorrelation function corresponding to the same night

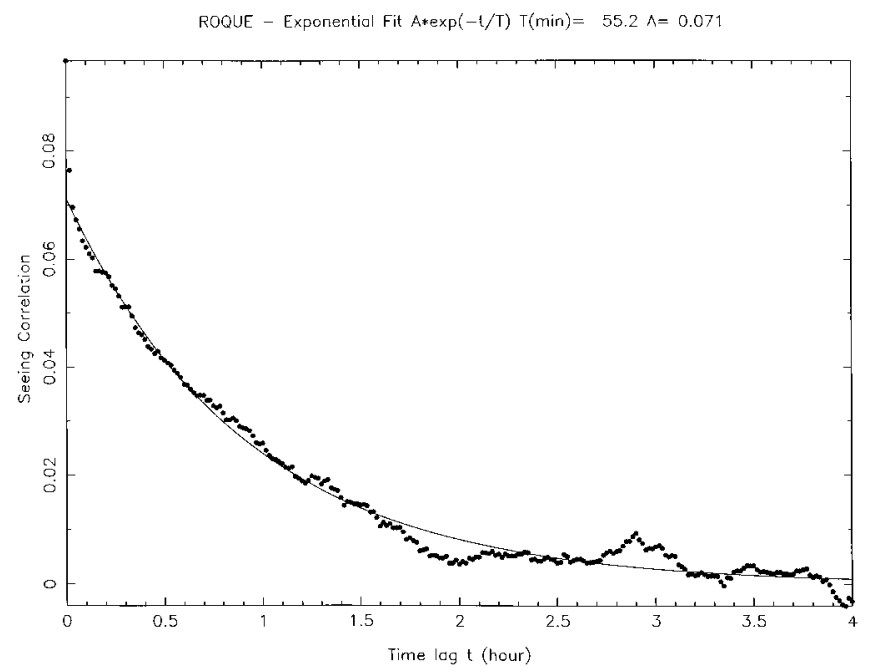

Fig. 10. Averaged autocorrelation function considering all the measurements. Superimposed, with a continuous line, is the best fit

\section{Summary and final remarks}

Nine months' statistics of seeing at the ORM, at two different sites, are presented. Measurements were taken on a 5-m tower to avoid spurious turbulence produced by the surface layer and with cross-calibrated seeing monitors. From the result of this monitoring, taken at a site near the location of the TNG with the DA/IAC DIMM, the excellence of the site, in terms of image quality is confirmed. Median seeing value of $0.64^{\prime \prime}$ was achieved over the whole period, with seeing values below $0.3^{\prime \prime}$ during $7 \%$ of the observing hours and minimum values down to $0.17^{\prime \prime}$. The total percentage of observed time with the DIMM is $72 \%$, well in agreement, within the limitations of opera- tion of our experiment, with the $79 \%$ that is reported by Carlsberg Meridian Circle, over several years.

Our routine observations combined with long temporal series meteorological data from the literature, allow us to identify June to September as the best months coinciding with the period of the year in which the strength of the temperature inversion layer is larger, due to the prevailing trade-wind regime. During this period, the height of the inversion layer $(\approx 1200 \mathrm{~m})$ is also lower. The existence of a seasonal dependence of seeing is then confirmed, with median values from $0.54^{\prime \prime}$ in summer and $0.77^{\prime \prime}$ during the rest of the year, most probably associated with the general climatology of the Canary Islands. Within this best period, it is worth mentioning the statistics corresponding to the month of June, with $0.46^{\prime \prime}$ median seeing value (0.17" rms).

Although during the last years a very big effort has been made in site characterization programmes, little long-term climatology is available to compare with the data presented here. Very good quality sites as Hawaii and Mount Graham, for instance, have never been checked routinely using cross-calibrated seeing monitors. The largest database has been established by ESO at La Silla and Paranal, developing a very extensive and reliable seeing monitoring. From the comparison between ESO (Sarazin 1995) and our data, presented in this paper, the three places, La Silla, Paranal and ORM are excellent, ORM presenting better behaviour, with the best median values, and a slightly larger percentage of seeing values below $0.3^{\prime \prime}$. The parameters corresponding to the best month are also better.

The comparison of two different areas at the ORM shows almost the same trend although the TNG slope is slightly better than the WHT slope during the summer period. This fact might be attributed to local disturbances 
of the WHT dome and terrace. Hence, no obvious change in the spatial localization of the surface-layer turbulence has been detected.

We have analysed the the temporal variation of the seeing in order to have a better insight into the processes which give rise to the optical turbulence. We found that during relatively stable nights, with about $0.5^{\prime \prime}$ mean typical values, the seeing can deteriorate abruptly over short periods. This can be interpreted by saying that even under good seeing conditions, and hence quasi-laminar flow, the steady equilibrium can be broken by a perturbation and degenerates into turbulence within a few minutes. This process is not reversible, and therefore we never observed a rapid seeing improvement. Another pattern that may appear sporadically is an oscillatory behaviour in the seeing temporal evolution during a given night. This periodical variation has a typical time scale of $45 \mathrm{~min}$, which falls within the buoyancy range of gravity waves. We computed an averaged autocorrelation function over all the available data during the nine months. No oscillations seem to remain in the correlation, and therefore a large spectrum of gravity waves is present in the atmosphere.

From the analysis of this autocorrelation function it is concluded a 1.2-hour exponential decay time of the optical turbulence.

Since October 1996, a site-testing programme led by the IAC to select the site for the future GTC has been under way. Data are taken simultaneously at two sites at the ORM. At each site we have installed a DIMM $(5 \mathrm{~m}$ above the ground) and a 12-m meteorological mast. These will provide a large and updated database on both optical quality and meteorology at the ORM. We will then be able to begin a study the relationship between seeing and meteorology.

Acknowledgements. This work has been funded by the Instituto de Astrofísica de Canarias and the programme for collaborative research between France and Spain (Acciones Integradas 1990, 1991, 1992). We are grateful to Sergio Ortolani and Dario Mancini from the Galileo group, who provided the 5-m tower, as well as the design of the foldable dome to protect the DIMM. Also their support and comments are acknowledged. Our observers, Amadeo Jorge Martín and Carlos González Suárez, have performed a very good job and their dedication is truly appreciated. The Site characterization project was promoted by Francisco Sanchez, to whom we are grateful also for valuable comments on this paper. Communication with the Isaac Newton Group on this topic has always been very good, and we are grateful to Mike Breare and Steve Unger for making it possible. We would also like to thank the staff of the ORM for ther help and in particular, Mary Barreto, the then Site Administrator of the ORM, for making things run smoothly. We thank Terry Mahoney for correcting the English of this paper. Many thanks are also due to our referee, Marc Sarazin, for his prompt revision of the paper.

\section{References}

Ardeberg A., 1984, in Proc. ESO Workshop No. 18, Site Testing for Future Large Telescopes, Ardeberg A. \& Wojter L. (eds.). Garching, ESO, p. 73

Azouit M., Vernin J., 1980, J. Atm. Sci. 37, 1550

Brandt P.N., Righini A., 1985, Vistas 28, 437

Coulman C., 1985, ARA\&A 23, 19

Coulman C.E., Vernin J., Fuchs A., 1995, Appl. Opt. 34, 5461

Cuevas E., 1995, PhD Thesis, Universidad Complutense de Madrid

Cuevas E., et al., 1996 (in preparation)

Dierickx P., 1992, Optical performances of large ground-based telescopes; J. Mod. Opt. 39, 569

Font-Tullot I., 1956, The weather in the Canary Islands (in Spanish) Madrid, Servicio Nacional de Meteorología, Publ. Ser. A 26

Fried D.L., 1966, J. Opt. Soc. Am. 56, 1372

Huetz de Lemps A., 1969, The Climate of the Canary Islands (in French) Paris, Faculté de Lettres et Sciences humaines de Paris-Sorbonne, Série "Recherches", Tome 54

Lee Y.W., 1960, Statistical Theory of Communication. New York, Wiley

Mancini D., Mancini G., Ortolani S., Zitelli V., 1993, The seeing monitor tower, TNG Technical Report No. 28

Martin F., Tokovinin A., Agabi A., Borgnino J., Ziad A., 1994, A\&AS 108, 173

Murdin P., 1985, Vistas 28, 449

Muñoz-Tuñón C., Fuentes F.J., 1990, Astrophys. Sp. Sci. 171, 257

Pike C.D., 1984, La Palma Tech. Note

Rodrìguez-Espinosa J.M., Alvarez P., 1996, Proc. ESO/SPIE Conference, Telescopes of Today and Tomorrow, Ardeberg A. (ed.) (in press)

Roddier C., 1976, J. Opt. Soc. Am. 66, 478

Rösch J., 1963, IAU Symp. 19, Le choix des sites d'observatoires astronomiques

Sánchez F., 1970, Urania 271-272, 97

Sarazin M., Roddier F., 1990, A\&A 227, 294

Sarazin M., 1995, ESO Technical Note on Site Quality Assessment: Observatorio del Roque de los Muchachos, La Palma, Islas Canarias

Vernin J., Muñoz-Tuñón C., 1992, A\&A 257, 811

Vernin J., Muñoz-Tuñón C., 1994, A\&A 284, 311

Vernin J., Muñoz-Tuñón C., 1995, PASP 107, 1

Stock J., Keller G. 1960, in Stars and Stellar Systems, Vol. 1: Telescopes, Kuiper G.P. \& Middlehurst B.M. (eds.). Chicago, University of Chicago Press, p. 138 